\title{
Overexpression of uncoupling protein 3 in skeletal muscle protects against fat-induced insulin resistance
}

\author{
Cheol Soo Choi, ${ }^{1}$ Jonathan J. Fillmore, ${ }^{1}$ Jason K. Kim, ${ }^{1}$ Zhen-Xiang Liu, ${ }^{1}$ Sheene Kim, ${ }^{1}$ \\ Emily F. Collier, ${ }^{1}$ Ameya Kulkarni, ${ }^{1}$ Alberto Distefano, ${ }^{1}$ Yu-Jin Hwang, ${ }^{1}$ Mario Kahn, ${ }^{1}$ Yan Chen, ${ }^{2}$ \\ Chunli Yu, ${ }^{1}$ Irene K. Moore, ${ }^{1}$ Richard M. Reznick, ${ }^{1}$ Takamasa Higashimori, ${ }^{1}$ and Gerald I. Shulman ${ }^{1,2,3}$ \\ 1Department of Internal Medicine, ${ }^{2}$ Department of Cellular and Molecular Physiology, and \\ ${ }^{3}$ Howard Hughes Medical Institute, Yale University School of Medicine, New Haven, Connecticut, USA.
}

\begin{abstract}
Insulin resistance is a major factor in the pathogenesis of type 2 diabetes and is strongly associated with obesity. Increased concentrations of intracellular fatty acid metabolites have been postulated to interfere with insulin signaling by activation of a serine kinase cascade involving PKC $\theta$ in skeletal muscle. Uncoupling protein 3 (UCP3) has been postulated to dissipate the mitochondrial proton gradient and cause metabolic inefficiency. We therefore hypothesized that overexpression of UCP3 in skeletal muscle might protect against fat-induced insulin resistance in muscle by conversion of intramyocellular fat into thermal energy. Wild-type mice fed a high-fat diet were markedly insulin resistant, a result of defects in insulin-stimulated glucose uptake in skeletal muscle and hepatic insulin resistance. Insulin resistance in these tissues was associated with reduced insulin-stimulated insulin receptor substrate 1- (IRS-1-) and IRS-2-associated PI3K activity in muscle and liver, respectively. In contrast, UCP3-overexpressing mice were completely protected against fat-induced defects in insulin signaling and action in these tissues. Furthermore, these changes were associated with a lower membrane-to-cytosolic ratio of diacylglycerol and reduced PKC $\theta$ activity in whole-body fat-matched UCP3 transgenic mice. These results suggest that increasing mitochondrial uncoupling in skeletal muscle may be an excellent therapeutic target for type 2 diabetes mellitus.
\end{abstract}

\section{Introduction}

The association of obesity with insulin resistance and type 2 diabetes is well established, and today's high-fat Western diet and increased sedentary lifestyle have made type 2 diabetes a worldwide epidemic (1). Development of insulin resistance has been associated with alterations in lipid metabolism and increased intracellular lipid content in skeletal muscle and liver (2-13). Weight loss is an important therapeutic intervention in diabetic patients $(14,15)$. However, weight loss strategies including diet, exercise, and pharmacologic tools have had only short-term success and are associated with a 95\% failure rate (16). A long-term strategy might involve increasing patients' metabolic rates by decreasing their metabolic efficiency (17-19). Uncoupling proteins (UCPs) are inner mitochondrial membrane transporters that dissipate the mitochondrial proton gradient, thus uncoupling oxidative phosphorylation. Stored energy is therefore expended as heat. UCP1, the first UCP characterized, is expressed exclusively in brown adipose tissue, which is important in regulating body temperature and body weight in rodents $(20,21)$. Moreover, UCP1-deficient mice have increased susceptibility to diet-induced obesity with age (22), and ectopic brown adipose tissue in muscle of 129S6/SvEvTac mice protects them from diet-induced weight gain (18). However, the contribution of brown adipose tissue to body tempera-

Nonstandard abbreviations used: DAG, diacylglycerol; HFD, high-fat diet; IRS, insulin receptor substrate; LPA, lysophosphatidic acid; RC, regular chow; UCP, uncoupling protein.

Conflict of interest: The authors have declared that no conflict of interest exists. Citation for this article: J. Clin. Invest. 117:1995-2003 (2007). doi:10.1172/JCI13579. ture and body weight regulation is not believed to play a significant role in adult humans. UCP2 and UCP3 were recently identified with high sequence homology to UCP1 (23-25). Their physiological roles still remain unclear, although UCP3 has been suggested to be important in 3,4-methylenedioxymethamphetamine-induced thermogenesis (19). Evidence suggests that UCP2 and UCP3 may not mediate adaptive thermogenesis: $U C P 2$ and UCP 3 mRNA and protein expression increase in muscle of starved rats, whereas thermogenesis decreases (26-29), and UCP2 knockout and UCP3 knockout mice have normal responses to cold exposure and are not obese (30-33). UCP3 is highly expressed in skeletal muscle in humans and rodents (34). While UCP3 expression in muscle is only $0.1 \%$ to $1 \%$ of UCP 1 expression in brown adipose tissue (35), it is possible that even this low expression of UCP3 could still have substantial contributions to intramyocellular lipid metabolism and whole-body energy metabolism, considering the large size of the muscle mass. For example, UCP3 expression is upregulated by food deprivation and dietary fat, suggesting a role for UCP3 in the regulation of intracellular lipid metabolism and energy expenditure $(29,36)$, and transgenic mice with overexpression of UCP3 in muscle were found to be lean despite being hyperphagic (37). In addition, UCP3 transgenic mice displayed reduced fasting plasma glucose and insulin concentrations, which suggests that these mice may be more insulin sensitive than their wild-type littermates. Because accumulation of intramyocellular triglycerides $(3,38-40)$ or fatty acid metabolites $(4,10-13,41,42)$ has been implicated in the pathogenesis of insulin resistance, we hypothesized that overexpression of UCP3 in skeletal muscle might protect these mice against fat-induced insulin resistance by promoting the 


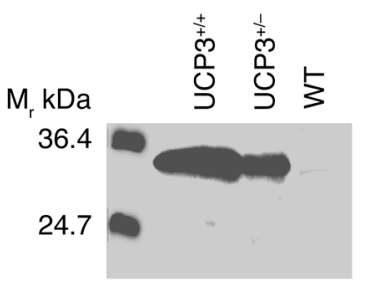

Figure 1

Western blot analysis. Human UCP3 expression at molecular mass 34,000 in the mixed gastrocnemius mitochondrial fraction of homozygous UCP3 transgenic mice, heterozygous UCP3 transgenic mice bred with B6CBAF1/J mice, and control wild-type B6CBAF1/J mice. Lane 1, molecular markers.

conversion of intracellular fat into heat. In order to test this hypothesis and develop a better understanding of the mechanism of fat-induced insulin resistance, we examined whole-body energy expenditure and insulin action in liver and muscle tissue by hyperinsulinemic-euglycemic clamp in awake UCP3 transgenic mice and age-matched wild-type mice fed either regular chow (RC) or high-fat diet (HFD). In order to match body fat composition in the 2 groups of mice, we performed additional studies in young (6-week-old) HFD-fed UCP3 transgenic mice that were matched for percent body fat with age-matched wildtype control mice.

\section{Results}

\section{UCP3 transgenic mice with mixed B6CBA background}

UCP3 Western blot analysis. A detailed analysis of the creation, tissue-specific mRNA expression, and protein expression of UCP3 transgenic mice was previously published (37). Figure 1 shows levels of human UCP3 overexpression in homozygous UCP3 transgenic mice bred on a B6CBAF1/J background, heterozygous UCP3 transgenic mice bred with $\mathrm{B} 6 \mathrm{CBAF} 1 / \mathrm{J}$ mice, and control wild-type B6CBAF1/J mice. The levels of UCP3 expression in the homozygotic mice increased 2.3-fold more than those of heterozygous mice and 60-fold more than those of control wild-type B6CBAF1/J mice, consistent with previous findings in these mice (37).

Weight gain and fat composition. The HFD-fed wild-type mice had a 2-fold higher weight gain compared with wild-type mice fed RC (26\% increase versus $12 \%$ increase), while there was no difference in weight gain in the UCP3 transgenic mice fed RC versus HFD (13\% increase; Figure 2). The body fat composition of the RC-fed UCP3 transgenic mice was significantly lower than that of wild-type mice fed the same diet (Table 1). UCP3 transgenic mice fed HFD also gained less fat mass ( $31 \%$ increase) than did wild-type mice $(81 \%$ increase) fed the HFD (Table 1).

Hepatic and peripheral insulin sensitivity. Basal concentrations of insulin and glucose were not significantly different between the groups of mice (Table 1). During the clamps, plasma insulin concentrations were raised to approximately $800 \mathrm{pM}$ and plasma glucose levels were maintained at approximately $7.5 \mathrm{mM}$ in all groups with a variable infusion of glucose. The glucose infusion rate needed to maintain euglycemia in all groups of mice reached a steady state within 30 minutes. The glucose infusion rate in the HFD-fed wild-type group required to maintain euglycemia was $60 \%$ lower compared to RC-fed wild-type mice (Figure 3A), indicating marked whole-body insulin resistance in this group.
In contrast, the HFD had no effect on the glucose infusion rate in UCP3 transgenic mice.

High-fat feeding decreased the rate of insulin-stimulated wholebody glucose uptake by $33 \%$ in the wild-type mice (Figure 3B). In contrast, the HFD had no effect on insulin-stimulated wholebody glucose uptake in the UCP3 transgenic mice. Insulinstimulated rates of whole-body glycolysis (Figure 3C) and glycogen/lipid synthesis (Figure 3D) in the HFD-fed wild-type group decreased by $47 \%$ and $37 \%$, respectively, compared with RC-fed wild-type mice. In contrast, insulin-stimulated whole-body glycolysis and glycogen/lipid synthesis in the HFD-fed UCP3 transgenic mice remained normal.

In addition, high-fat feeding caused hepatic insulin resistance in wild-type mice, as reflected by insulin's inability to suppress basal endogenous glucose production (Figure 4A). Surprisingly, UCP3 overexpression in skeletal muscle also protected the liver from fat-induced insulin resistance, as reflected by normal insulin suppression of endogenous glucose production (Figure 4A). While the hepatic insulin resistance in HFD-fed wild-type mice corresponded to a $36 \%$ decrease in hepatic insulin receptor substrate-2-associated (IRS-2-associated) PI3K activity, the HFDfed UCP3 transgenic mice had normal IRS-2-associated PI3K activity (Figure 4B).

The decrease in insulin-stimulated whole-body glucose uptake in the HFD-fed wild-type mice was a result of a $52 \%$ decrease in insulin-stimulated glucose uptake in skeletal muscle, as assessed by measuring 2 -deoxy-D-[1- $\left.{ }^{14} \mathrm{C}\right]$ glucose (Figure $5 \mathrm{~A}$ ). Insulin-stimulated rates of skeletal muscle glycolysis and glycogen synthesis were also decreased by $48 \%$ and $76 \%$, respectively (Figure 5, B and C). In contrast, UCP3 transgenic mice were protected against any fatinduced defects in insulin-stimulated rates of glucose uptake and metabolism in skeletal muscle. The decreased insulin-stimulated glucose uptake and metabolism seen in the skeletal muscle of HFDfed wild-type mice was associated with a $41 \%$ decrease in IRS-1associated PI3K activity (Figure 5D). In contrast, HFD-fed UCP3 transgenic mice had normal insulin-stimulated muscle IRS-1associated PI3K activity (Figure 5D), consistent with the findings of normal insulin action on glucose transport, glycolysis, and glycogen synthesis in skeletal muscle in these mice.

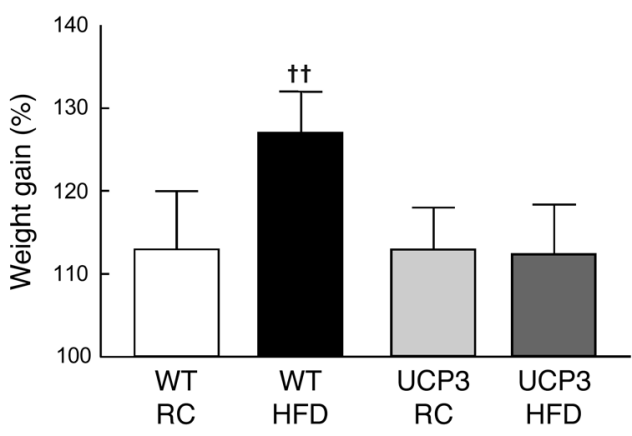

\section{Figure 2}

Weight gain for wild-type and UCP 3 transgenic mice on RC or HFD. $n=10$ (WT RC, WT HFD); 11 (UCP3 RC); 13 (UCP3 HFD). Weekly weights were assessed over the course of 4 weeks. The weight of RC-fed wildtype mice increased $13 \% \pm 6 \%$, while that of HFD-fed wild-type mice increased $27 \% \pm 6 \%$, representing a 2 -fold higher rate of weight gain. In contrast, there was no difference in the rate of weight gain in the UCP3 transgenic mice fed $\mathrm{RC}$ versus HFD. ${ }^{\dagger+} P=0.02$ versus all other groups. 
Table 1

Whole-body fat compositions and plasma concentrations of metabolites and hormones in wild-type and UCP3 transgenic mice with mixed background

\begin{tabular}{lcccc} 
& $\begin{array}{c}\text { RC-fed } \\
\text { wild type } \\
(\boldsymbol{n}=\mathbf{1 0})\end{array}$ & $\begin{array}{c}\text { HFD-fed } \\
\text { wild type } \\
(\boldsymbol{n}=\mathbf{1 0})\end{array}$ & $\begin{array}{c}\text { RC-fed } \\
\text { UCP3 } \\
(\boldsymbol{n}=\mathbf{1 1})\end{array}$ & $\begin{array}{c}\text { HFD-fed } \\
\text { UCP3 } \\
(\boldsymbol{n}=\mathbf{1 0})\end{array}$ \\
Glucose (mM) & $8.7 \pm 0.5$ & $9.9 \pm 0.6$ & $8.4 \pm 0.3$ & $8.0 \pm 0.6$ \\
Insulin (pM) & $47 \pm 5$ & $62 \pm 6$ & $44 \pm 9$ & $53 \pm 6$ \\
Fatty acids (mM) & $0.9 \pm 0.2$ & $1.2 \pm 0.1$ & $0.7 \pm 0.2$ & $0.8 \pm 0.1$ \\
Leptin (nM) & $2.9 \pm 0.4$ & $13.1 \pm 0.6^{A}$ & $3.6 \pm 0.6$ & $3.8 \pm 0.4$ \\
Fat mass (\% body mass) & $19.2 \pm 0.9$ & $34.7 \pm 1.0^{\mathrm{A}}$ & $15.1 \pm 1.0^{\mathrm{B}}$ & $20.2 \pm 0.8$ \\
\hline
\end{tabular}

${ }^{A} P<0.001$ versus other groups. ${ }^{B} P=0.02$ versus $R C$-fed wild type.

\section{Age- and fat-matched wild-type and UCP3 transgenic mice}

In order to match the ratio of fat to whole-body mass in wild-type and UCP3 transgenic mice and to exclude the confounding effect of mixed background of the mice on glucose and fat metabolism, 6-week-old, inbred (backcrossed more than 9 generations) wildtype mice and homozygous UCP3 transgenic mice were fed HFD for 10 days. Studying the mice at this early age resulted in no significant difference in body fat percentage between the 2 groups, as assessed by nuclear magnetic resonance analysis (wild-type, $12.4 \% \pm 0.6 \%$; UCP3 $11.9 \% \pm 0.5 \%$; NS). In order to further evaluate the prevention of HFD-induced obesity in UCP3 transgenic mice, energy balance of these mice was assessed in an animal metabolic monitoring system for 4 days ( 2 days for acclimation followed by 2 days of measurements). Although locomotor activity was similar in the wild-type and UCP3 transgenic mice, total energy expenditure and food intake were $30 \%$ and $50 \%$ higher, respectively, in UCP3 transgenic mice than in age- and fat-matched wild-type mice (Figure 6, A-C). Despite the increased energy expenditure and food intake in UCP3 transgenic mice, there were no differences in the respiratory quotient between the 2 groups (Figure 6D).

Peripheral insulin responsiveness is increased in UCP3 transgenic mice. Fasting plasma glucose and insulin concentrations were reduced by $23 \%$ and $40 \%$, respectively, in the UCP3 transgenic mice, whereas plasma fatty acid concentrations were similar in both groups in the fasting and clamp periods (Table 2). To gain further insight into insulin action on whole-body and tissuespecific glucose metabolism, we performed hyperinsulinemic-euglycemic clamps with radioisotope-labeled glucose infusion.

Despite similar whole-body fat ratios, UCP3 transgenic mice were protected against HFD-induced insulin resistance, as reflected by a $130 \%$ higher glucose infusion rate required to maintain euglycemia in UCP3 transgenic mice compared with fat-matched wild-type mice (Figure 7A). This increase in glucose infu-
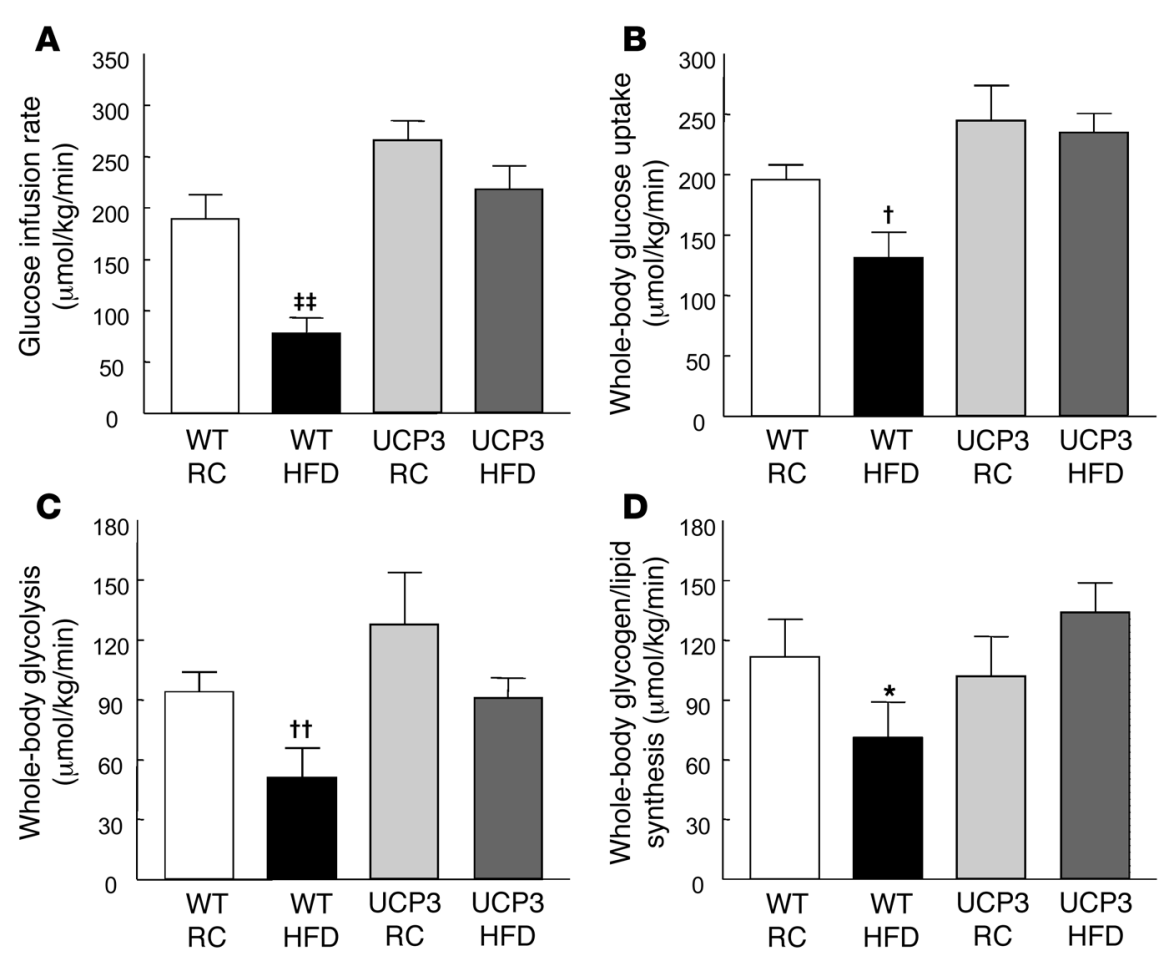
implicated increased novel PKC activity in muscle (PKC $\theta$ ) and liver (PKC 8 ) in mediating fat-induced insulin resistance in these tissues $(2,10-13,42,43)$. Consistent with this hypothesis, improved insulin sensitivity in muscle in the UCP3 transgenic mice was associated with a $60 \%$ increase in insulin-stimulated Akt2 activity (Figure 8B) and a 90\% decrease in PKC $\theta$ activity in muscle (Figure 8C).

Intracellular fat metabolites in skeletal muscle. Intracellular concentrations of triglyceride and lysophosphatidic acid (LPA) were similar in the skeletal muscle of UCP 3 transgenic mice compared with wild-type mice, although the long-chain fatty acyl-CoA concentration was higher in UCP3 transgenic mice (Figure 8, D, E, and G). However, the membrane-to-cytosolic ratio of diacylglycerol

Sion rate corresponded to a $70 \%$ increase in insulin-
stimulated whole-body glucose uptake in the UCP3 transgenic mice (Figure 7B), while insulin-stimunot different (UCP3, 31\% $\pm 9 \%$; wild-type, 13\% $\pm 9 \%$; NS). Insulin-stimulated whole-body glycolysis (Figure 7C) and whole-body glycogen/lipid synthesis
(Figure 7D) in the UCP3 transgenic mice increased by $62 \%$ and $86 \%$, respectively. The increase in insulinstimulated whole-body glucose uptake seen in the increase in insulin-stimulated glucose uptake in skeletal muscle (Figure 8A).

Increased Akt2 activity and decreased PKC $\theta$ in skeletal muscle of UCP 3 transgenic mice. We have previously 

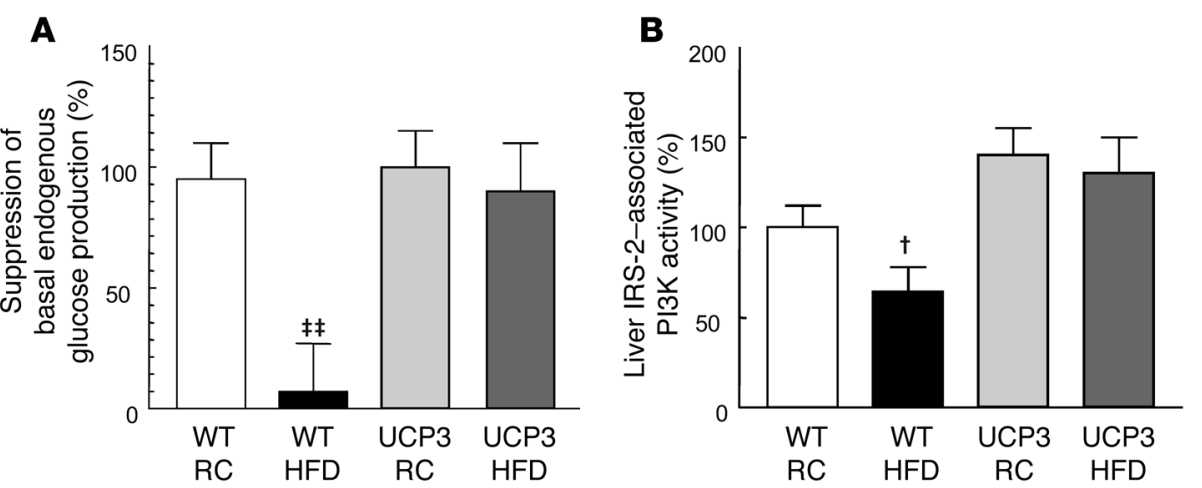

\section{Figure 4}

Endogenous glucose production and insulin signaling in liver during hyperinsulinemic-euglycemic clamps for wild-type and UCP3 transgenic mice on RC or HFD. $n=8$ (WT RC, UCP3 HFD); 10 (UCP3 RC); 11 (WT HFD). (A) Percent suppression of basal endogenous glucose production. $\sharp \ddagger P<0.001$ versus all other groups. (B) IRS-2-associated PI3K activity. ${ }^{\dagger} P=0.05$ versus all other groups.
(DAG), a known activator of PKC $\theta$, was $21 \%$ lower in UCP3 transgenic mice than in wild-type mice (Figure $8 \mathrm{~F}$ ), while intracellular ceramide concentration was higher in the UCP3 transgenic mice (UCP3, $61.0 \pm 2.3 \mathrm{nmol} / \mathrm{g}$; WT, $53.5 \pm 1.9 \mathrm{nmol} / \mathrm{g} ; P=0.02$ ).

AMP-activated protein kinase- $\alpha 2$ activity in skeletal muscle. AMPactivated protein kinase- $\alpha 2$ (AMPK- $\alpha 2$ ) activity was measured, because high levels of UCP3 expression in the homozygous UCP3 transgenic mice could result in lower ATP/AMP levels as a result of excessive mitochondrial uncoupling, leading to activation of AMPK in muscle and subsequent increased insulin sensitivity. However, there was no differences in AMPK- $\alpha 2$ activity in type 2 fiber muscle (extensor digitorum longus; wild-type, $0.30 \pm 0.06 \mathrm{pmol} / \mathrm{min} / \mathrm{mg}$; UCP3, $0.21 \pm 0.06$ $\mathrm{pmol} / \mathrm{min} / \mathrm{mg}$; NS), or mixed muscle (tibialis anterior; wild-type, $0.36 \pm 0.09 \mathrm{pmol} / \mathrm{min} / \mathrm{mg}$; $\mathrm{UCP} 3,0.27 \pm 0.05 \mathrm{pmol} / \mathrm{min} / \mathrm{mg}$; NS), between the 2 groups.

\section{Discussion}

In this study, we found that UCP3 overexpression in skeletal muscle protected mice from HFD-induced insulin resistance. In order to examine the mechanism by which this occurred, we performed hyperinsulinemiceuglycemic clamps to assess rates of insulinstimulated muscle and hepatic glucose metabolism. In wild-type mice, the HFD caused defects in insulin-stimulated whole-body glucose uptake and metabolism. Skeletal muscle glucose uptake is an important determinant for insulin-stimulated glucose utilization (44), and the decrease in whole-body glucose uptake and metabolism was associated with a decrease in insulin-stimulated glucose uptake in skeletal muscle. In contrast, UCP3 transgenic mice were protected against these fatinduced defects in insulin-stimulated muscle glucose metabolism. The HFD also caused marked hepatic insulin resistance, as reflected by insulin's inability to suppress endogenous glucose production in HFD-fed wild-type mice. Surprisingly, UCP3 overexpression in skeletal muscle also protected against fatinduced insulin resistance in the liver, which can likely be attributed to the reduced whole- body fat mass and decreased hepatic steatosis in the UCP3 transgenic mice caused by increased whole-body energy expenditure.

To exclude the effects of differences in the amount of wholebody fat mass and possible differences in genetic background, we performed additional experiments using young (6-week-old) UCP3 transgenic mice that were extensively backcrossed (more than 9 generations) and wild-type mice, all of which were fed HFD for 10 days. With this strategy, we were able to match the body fat percentage in these 2 groups of mice. Despite similar percent fat mass in these groups, we found that the wild-type mice were severely insulin resistant following high-fat feeding for 10 days, whereas the UCP3 transgenic mice were still protected from this
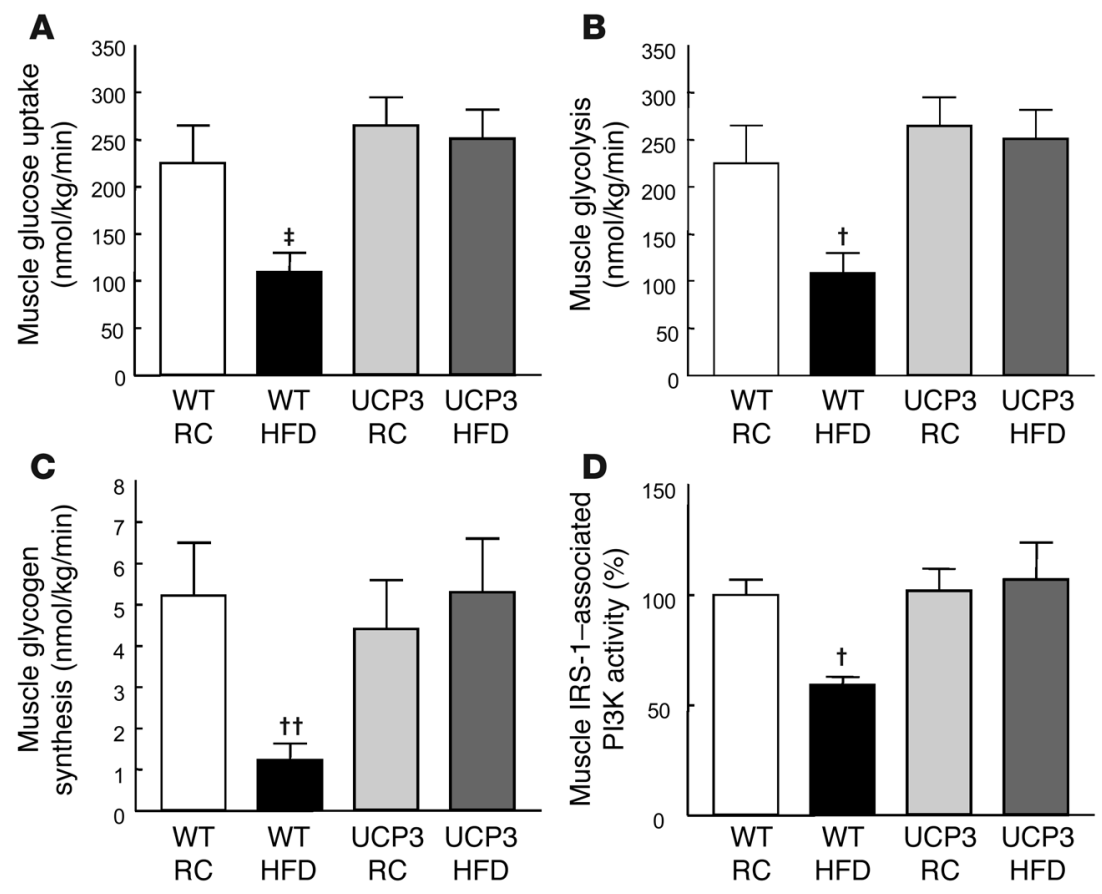

\section{Figure 5}

Skeletal muscle (gastrocnemius) metabolic parameters and insulin signaling during hyperinsulinemic-euglycemic clamps for wild-type and UCP3 transgenic mice fed RC or HFD. $n=8$ (WT RC, UCP3 HFD); 10 (UCP3 RC); 11 (WT HFD). (A) In vivo insulin-stimulated glucose uptake in skeletal muscle. $¥ P<0.01$ versus all other groups. (B) In vivo insulinstimulated rate of skeletal muscle glycolysis. ${ }^{\dagger} P=0.05$ versus all other groups. (C) In vivo insulin-stimulated rate of skeletal muscle glycogen synthesis. ${ }^{t \dagger} P=0.02$ versus all other groups. (D) IRS-1-associated PI3K activity. ${ }^{\dagger} P=0.05$ versus all other groups. 

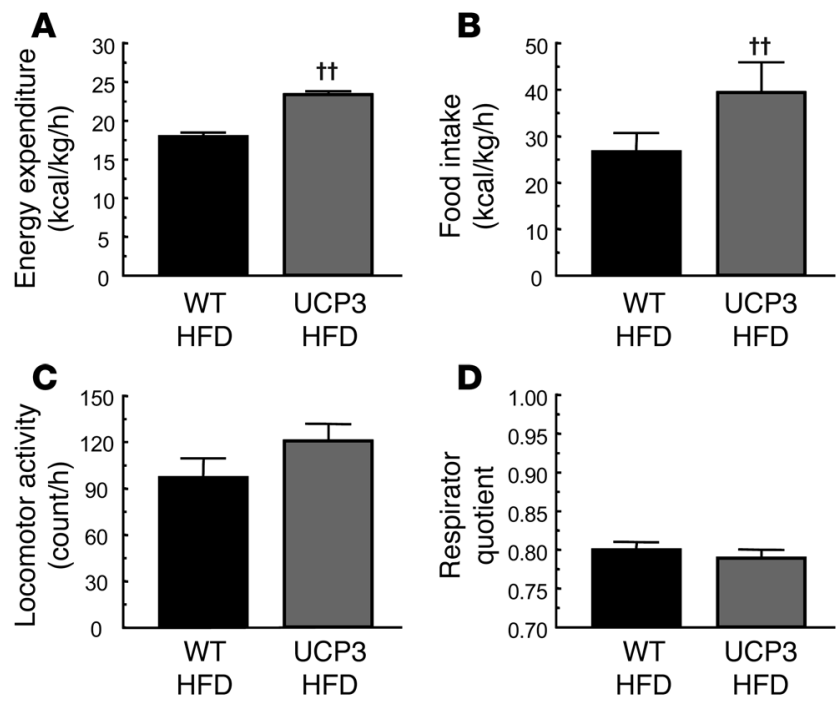

Figure 6

Energy balance and substrate selection. $n=8$ per group. Energy expenditure (A), food intake (B), locomotor activity (C), and respiratory quotient (D) were assessed by a comprehensive animal metabolic monitoring system in fat-matched wild-type and UCP3 transgenic mice fed HFD for 10 days. Data are mean \pm SEM. ${ }^{\dagger \dagger} P<0.05$ versus wild type.

fat-induced insulin resistance and continued to show normal whole-body and muscle insulin sensitivity.

The mechanism by which fat causes insulin resistance remains controversial. Randle and coworkers first demonstrated that fatty acids compete with glucose for oxidation in isolated rat heart and diaphragm muscle, and therefore hypothesized that increased fat oxidation might cause the insulin resistance associated with diabetes and obesity (45). The proposed mechanism was that an increase in fatty acids caused an increase in the intramitochondrial acetyl $\mathrm{CoA} / \mathrm{CoA}$ and $\mathrm{NADH} / \mathrm{NAD}+$ ratios, with subsequent inactivation of pyruvate dehydrogenase activity (45). This in turn would cause intracellular concentrations of citrate to increase, leading to allosteric inhibition of phosphofructokinase activity. Subsequent increases in glucose-6-phosphate concentration would lead to inhibition of hexokinase activity, resulting in decreased glucose uptake. Recent studies by our group have suggested a very different mechanism for fatinduced insulin resistance in skeletal muscle $(2,42,43,46,47)$. We hypothesized that fatty acids directly interfere with insulin activation of glucose transport in skeletal muscle by inhibiting insulin activation of IRS-1-associated PI3K activity $(2,4,8,43$, 46, 48). Studies in Irs1-disrupted mice have shown that IRS-1 is an important component of the insulin signaling pathway, leading to activation of glucose transport and glycogen synthesis in skeletal muscle $(49,50)$. The observed defects in fat-induced, insulin-stimulated glucose uptake in the muscle of HFD-fed wild-type mice were associated with decreased activity of IRS-1associated PI3K activity in muscle. These findings are consistent with our previous observations of lipid-induced insulin resistance in mice, rats, and humans $(4,8,42,43,46)$. In contrast, UCP3-overexpressing mice were completely protected from HFD-induced alteration in insulin signaling of skeletal muscle. The mechanism for this decreased activity in the skeletal muscle of HFD-fed wild-type mice might involve serine phosphoryla- tion of IRS- 1 through a serine phosphorylation cascade initiated by PKC $\theta(2,42,43,46)$. This serine phosphorylation would interfere with tyrosine phosphorylation of these substrates by the insulin receptor, leading to a decrease in IRS-1-associated PI3K activity $(42,51)$. In support of this hypothesis, we previously found that PKC $\theta$ knockout mice were protected from fat-induced insulin resistance during lipid infusion (52), and in the present study we found that UCP-overexpressing mice had significantly lower PKC $\theta$ activation in skeletal muscle compared with HFD-fed wild-type mice. Increased intracellular concentrations of fatty acyl-CoAs, DAG, LPA, and ceramides have all been proposed to be responsible for mediating fat-induced insulin resistance, and DAG is a known activator of PKC $\theta$ $(2,53-57)$. Here we showed that intramuscular DAG likely plays a major role in mediating the insulin resistance in skeletal muscle of wild-type mice, because the membrane-to-cytosolic ratio of DAG was increased in the muscle of wild-type mice compared with UCP3 transgenic mice. In contrast, there were no differences in the intramuscular concentrations of triglyceride or LPA between the groups. Moreover, intramuscular ceramide and fatty acyl-CoAs were markedly increased in the muscle of UPC3 transgenic mice. Taken together, these data demonstrate that fatty acyl-CoA, LPA, ceramide, and triglyceride are not the triggers for fat-induced insulin resistance in skeletal muscle in this mouse model.

Recently it was suggested that the increased metabolism and weight loss in UCP3-overexpressing mice is caused by the unphysiological coupling in mitochondrial proton conductance (58). The evidence supporting this claim includes an increase in uncoupling not proportional to the increase in UCP3 expression and uncoupling not being activated by superoxide or inhibited by GDP (58). However, superoxide, a physiological activator of UCPs, increased proton conductance in rat skeletal muscle, which correlated with doubling of native UCP3 protein $(59,60)$. Therefore, even if overexpressed UCP3 in the skeletal muscle of UCP3 transgenic mice uncouples differently than native UCP3 proteins, these data support the role of mitochondrial uncoupling in skeletal muscle as a potential strategy to increase energy expenditure and treat obesity and type 2 diabetes.

In summary, these data demonstrate that overexpression of UCP3 in skeletal muscle protects against fat-induced defects in insulin signaling and action in skeletal muscle, which may be mediated by a lower membrane-to-cytosolic ratio of DAG, leading to decreased PKC $\theta$ activation and increased insulin signal transduction at the level of IRS-1 tyrosine phosphorylation.

\section{Table 2}

Plasma concentrations of metabolites and hormones in 6-weekold wild-type and UCP3 transgenic mice fed HFD for 10 days

\begin{tabular}{lcccc} 
& \multicolumn{2}{c}{ Basal } & \multicolumn{2}{c}{ Clamp } \\
& Wild type & UCP3 & Wild type & UCP3 \\
Glucose (mM) & $9.8 \pm 0.6$ & $6.5 \pm 0.2^{\mathrm{A}}$ & $7.0 \pm 0.3$ & $7.3 \pm 0.1$ \\
Insulin (pM) & $113 \pm 8.4$ & $69 \pm 7.6^{\mathrm{B}}$ & $463 \pm 20$ & $419 \pm 27$ \\
Fatty acids (mM) & $1.65 \pm 0.2$ & $1.81 \pm 0.1$ & $1.17 \pm 0.18$ & $1.15 \pm 0.15$ \\
Resistin (nM) & $1.15 \pm 0.15$ & $1.04 \pm 0.18$ & ND & ND \\
Leptin (nM) & $1.4 \pm 0.4$ & $0.4 \pm 0.1$ & ND & ND \\
\hline
\end{tabular}

ND, not determined. $n=10-11$ per group. ${ }^{A} P<0.001$ versus wild type. B $P<0.05$ versus wild type. 
A
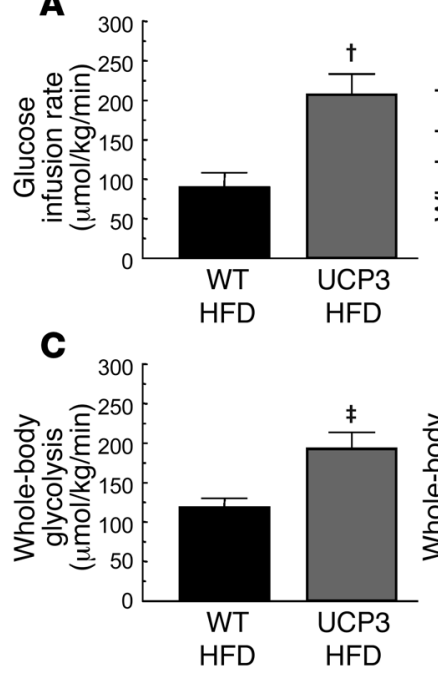
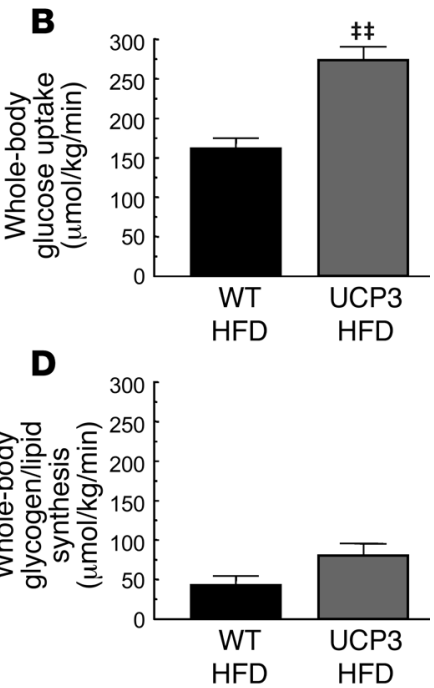

Figure 7

Whole-body metabolic parameters during hyperinsulinemic-euglycemic clamps for fat-matched wild-type and UCP3 transgenic mice fed HFD for 10 days. $n=10$ per group. (A) Steady-state glucose infusion rate. $\dagger P=0.002$ versus wild type. (B) Insulin-stimulated rate of whole-body glucose uptake. $\neq \neq P<0.001$ versus wild type. (C) Insulin-stimulated rate of whole-body glycolysis. $¥ P=0.006$ versus wild type. (D) Insulinstimulated rate of whole-body glycogen/lipid synthesis $(P=0.06)$.

\section{Methods}

Animals. Details of the generation of the B6CBAF1/J mice overexpressing human UCP3 in skeletal muscle (gift of J.C. Clapham, GlaxoSmithKline, Cambridge, United Kingdom) used in the experiments have been described previously (37). These 8- to 9-week-old UCP3 transgenic mice and agematched control wild-type B6CBAF1/J male mice (The Jackson Laboratory) were studied. To minimize environmental differences, mice were housed together for at least 1 month before each experiment. In order to generate inbred mice, B6CBAF1/J mice overexpressing human UCP3 were backcrossed 9 more times to C57BL/6J mice. These inbred mice were used for fat-matched study. They were fed ad libitum either RC ( $14 \%$ calories from fat, $60 \%$ calories from carbohydrate, $26 \%$ calories from protein; Prolab RMH 3000; Purina Mills Inc.) or HFD (55\% calories from fat, $24 \%$ calories from carbohydrate, $21 \%$ calories from protein; TD93075; Harlan Teklad). They were housed under controlled temperature $\left(23^{\circ} \mathrm{C}\right)$ and lighting (12-hour light/12-hour dark) with free access to water. The study was conducted at the NIH-Yale Mouse Metabolic Phenotyping Center. All procedures were approved by the Yale University Animal Care and Use Committee.

UCP3 Western blot analysis. Immunoblots for UCP3 content were performed on gastrocnemius mitochondrial fractions (61) in homozygous UCP3 transgenic mice, heterozygous UCP3 transgenic mice bred with B6CBAF1/J mice, and control wild-type B6CBAF1/J mice. Primary rabbit anti-human UCP3 antibodies (Alpha Diagnostic Inc.) and secondary anti-rabbit horseradish peroxidase antibodies (Promega) were used. Final images were obtained with an ECL detection kit (Amersham Pharmacia Biotech).

Basal metabolic study. Fat and lean body masses were assessed by ${ }^{1} \mathrm{H}$ magnetic resonance spectroscopy (Bruker BioSpin) on 6-week-old wild-type and UCP3 transgenic mice before and after 10 days of HFD feeding $(n=11$ per group). Lean and fat tissue masses were determined and expressed as percentages of total body mass.

A comprehensive animal metabolic monitoring system (CLAMS; Columbus Instruments) was also used to evaluate activity, food consump-

tion, and energy expenditure after 10 days of HFD feeding. Energy expenditure and food intake data were normalized with respect to body weight. Energy expenditure and respiratory quotient (RQ) were calculated from the gas exchange data as follows: energy expenditure $=(3.815+1.232 \times$ $\mathrm{RQ}) \times \mathrm{VO}_{2}$, where $\mathrm{RQ}$ is the ratio of $\mathrm{VCO}_{2}$ to $\mathrm{VO}_{2}$, which changes depending on the energy source the animal is using. When carbohydrates are the only substrate being oxidized, the RQ is 1.0 , and it is 0.7 when only fatty acids are oxidized. Activity was measured on $x$ and $z$ axes using infrared beams to count the number of beam breaks during the specified measurement period. Feeding was measured by recording the difference in the scale measurement of the center feeder from one time point to another. We studied 8 male mice per group.

Hyperinsulinemic-englycemic clamp study. Seven days prior to the hyperinsulinemic-euglycemic clamp studies, indwelling catheters were placed into the right internal jugular vein extending to the right atrium. After an overnight fast, $\left[3-{ }^{3} \mathrm{H}\right]$-glucose (HPLC purified; PerkinElmer) was infused at a rate of $0.05 \mu \mathrm{Ci} / \mathrm{min}$ for 2 hours to assess the basal glucose turnover. Following the basal period, hyperinsulinemic-euglycemic clamp was conducted for 120 minutes with a primed/continuous infusion of human insulin (105 pmol $/ \mathrm{kg}$ prime, $15 \mathrm{pmol} / \mathrm{kg} / \mathrm{min}$ infusion; Novo Nordisk) to raise plasma insulin within the physiological range. Blood samples $(10 \mu \mathrm{l})$ were collected at 10- to 20-minute intervals for the immediate measurement of plasma glucose, and $20 \%$ dextrose was infused at variable rates to maintain plasma glucose at basal concentrations $(\sim 6.7 \mathrm{mM})$. To estimate insulin-stimulated whole-body glucose fluxes, $\left[3-{ }^{3} \mathrm{H}\right]$-glucose was infused at a rate of $0.1 \mu \mathrm{Ci} / \mathrm{min}$ throughout the clamps and 2-deoxy-D-[1-14 C]glucose (PerkinElmer) was injected as a bolus at the seventy-fifth minute of the clamp to estimate the rate of insulinstimulated tissue glucose uptake as previously described (62). Blood samples $(10 \mu \mathrm{l})$ for the measurement of plasma ${ }^{3} \mathrm{H}$ and ${ }^{14} \mathrm{C}$ activities were taken at the end of the basal period and during the last 45 minutes of the clamp. Additional blood samples were obtained for the measurement of plasma insulin and free fatty acid concentrations at the end of the basal and clamp periods. At the end of the clamp, mice were anesthetized with pentobarbital sodium injection, and tissues were taken for biochemical measurements within 4 minutes. Each tissue was dissected out within 2 seconds of exposure, frozen immediately using liquid nitrogen-cooled aluminum blocks, and stored at $-80^{\circ} \mathrm{C}$ for subsequent analysis.

Glucose flux calculation. For the determination of plasma ${ }^{3} \mathrm{H}$-glucose, plasma was deproteinized with $\mathrm{ZnSO}_{4}$ and $\mathrm{Ba}(\mathrm{OH})_{2}$, dried to remove ${ }^{3} \mathrm{H}_{2} \mathrm{O}$, resuspended in water, and counted in scintillation fluid (Ultima gold; PerkinElmer) on a Packard liquid scintillation counter (Packard Instrument Co.). Rates of basal and insulin-stimulated whole-body glucose turnover were determined as the ratio of the $\left[3-{ }^{-3} \mathrm{H}\right]$-glucose infusion rate (disintegrations per minute; $\mathrm{dpm}$ ) to the specific activity of plasma glucose (i.e., $\mathrm{dpm} / \mathrm{mg}$ ) at the end of the basal period and during the final 30 minutes of the clamp experiment, respectively. Hepatic glucose production was determined by subtracting the glucose infusion rate from the total glucose appearance rate.

The plasma concentration of ${ }^{3} \mathrm{H}_{2} \mathrm{O}$ was determined by the difference between ${ }^{3} \mathrm{H}$ counts without and with drying. Whole-body glycolysis was calculated from the rate of increase in plasma ${ }^{3} \mathrm{H}_{2} \mathrm{O}$ concentration divided by the specific activity of plasma ${ }^{3} \mathrm{H}$-glucose, as previously described (63). Whole-body glycogen synthesis was estimated by subtracting whole-body glycolysis from whole-body glucose uptake, assuming that glycolysis and glycogen synthesis account for the majority of insulinstimulated glucose uptake.

For the determination of individual tissue glucose uptake, tissue samples were homogenized, and the supernatants were subjected to an ionexchange column to separate tissue ${ }^{14} \mathrm{C}-2$-DG-6-phosphate from 2-DG. Tissue glucose uptake was calculated from the area under the curve of 

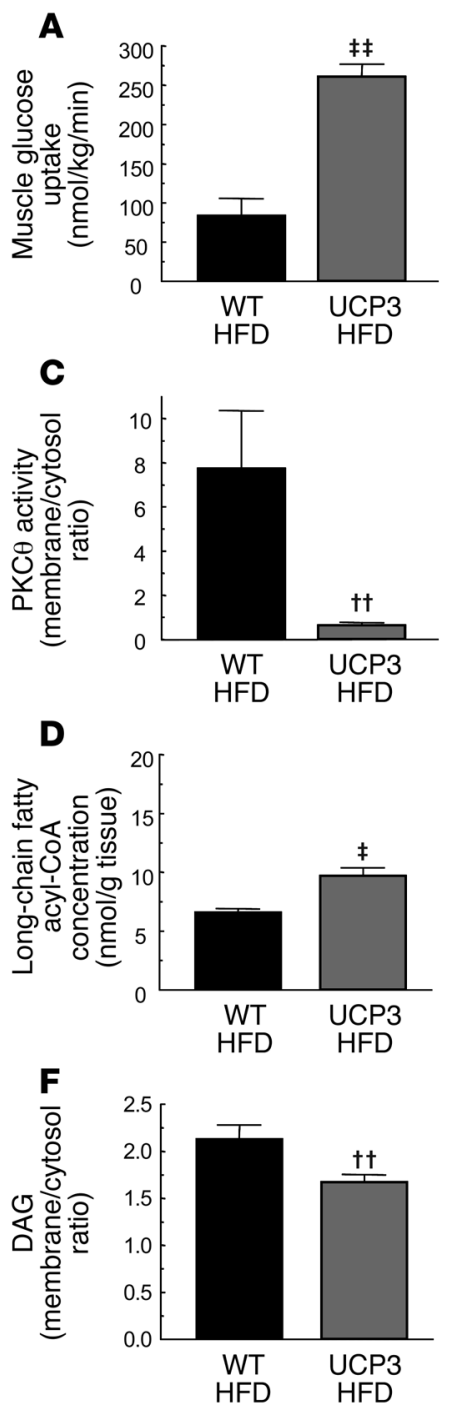
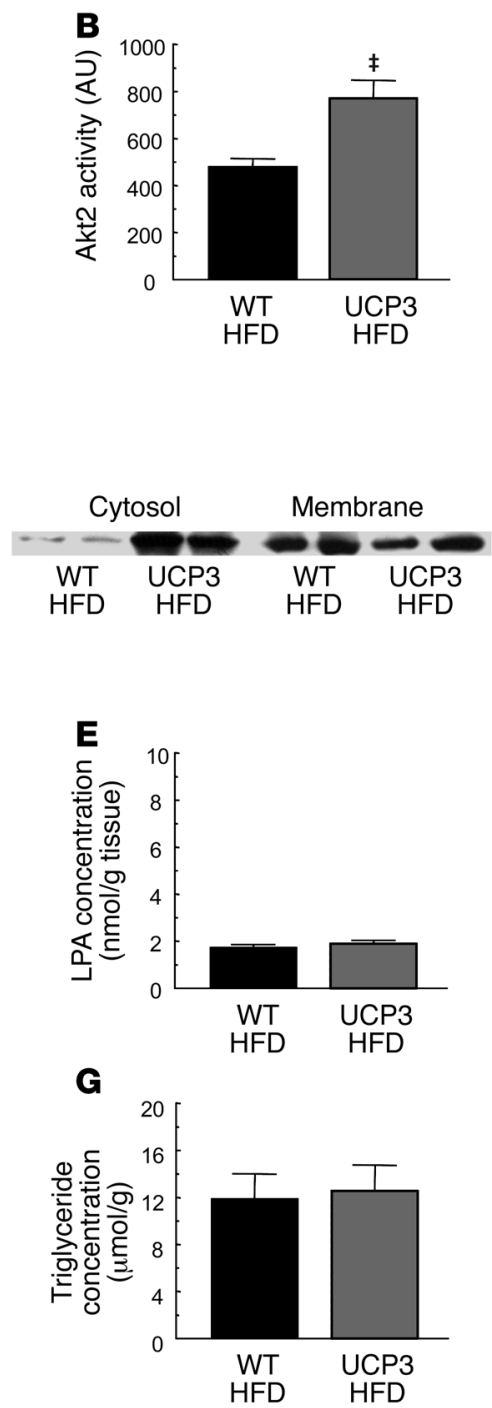

Figure 8

UCP3 overexpression increased glucose uptake and AKT activity and decreased PKC $\theta$ activity and membrane translocation of DAG in skeletal muscle of fat-matched wild-type and UCP3 transgenic mice fed HFD for 10 days. $n=9-10$ per group (A, B, and $\mathbf{D}-\mathbf{G})$; 4 per group (C). (A) In vivo insulin-stimulated glucose uptake. ${ }^{\ddagger \ddagger} P<0.001$ versus wild type. (B) In vivo insulin-stimulated Akt2 activity. $¥ P=0.002$ versus wild type. (C) PKC $\theta$ activity, as determined by the ratio of $\mathrm{PKC} \theta$ levels in the membrane fraction to those in the cytosolic fraction. ${ }^{\dagger+} P=0.02$ versus wild type. (D) Long-chain fatty acyl-CoA concentration. ${ }^{\ddagger} P=0.001$ versus wild type. (E) LPA concentration. (F) Membrane/cytosol ratio of DAG. ${ }^{\dagger+} P=0.02$ versus wild type. $(\mathbf{G})$ Triglyceride concentration.

the plasma ${ }^{14} \mathrm{C}-2-\mathrm{DG}$ profile and muscle ${ }^{14} \mathrm{C}-2$-DG-6-phosphate content, as previously described (63).

Biochemical analysis. Plasma glucose was analyzed during the clamps using $10 \mu \mathrm{l}$ plasma by a glucose oxidase method on a Beckman glucose analyzer II (Beckman). Plasma insulin levels were determined by RIA using a kit from Linco Research. Plasma fatty acid concentrations were determined using an acyl-CoA oxidase-based colorimetric kit (Wako). Plasma resistin and leptin concentrations were measured by multiplexed biomarker immunoassays (Lincoplex) from Linco Research.

Insulin signaling and PKCs. Akt2 activity was assessed in protein extracts from muscle harvested after short-term insulin stimulation. Akt2 assays were performed according to methods previously described (10, 64-66). The primary antibody used for experiments was rabbit polyclonal IgG; antibodies for Akt2 were obtained from Upstate. For PKC $\theta$ membrane translocation, $50 \mu \mathrm{g}$ of crude membrane and cytosol protein extracts were resolved by SDSPAGE using $8 \%$ gel and electroblotted onto polyvinylidene difluoride membrane (DuPont) using a semidry-transfer cell (Bio-Rad). The membrane was then blocked for 2 hours at room temperature in PBS-Tween $\left(10 \mathrm{mmol} / 1 \mathrm{NaH}_{2} \mathrm{PO}_{4} ; 80 \mathrm{mmol} / \mathrm{l}\right.$ $\mathrm{Na}_{2} \mathrm{HPO}_{4} ; 0.145 \mathrm{~mol} / 1 \mathrm{NaCl}$; and $0.1 \%$ Tween-20, pH 7.4) containing $5 \%(\mathrm{w} / \mathrm{v})$ nonfat dried milk, washed twice, and incubated overnight with rabbit anti-peptide antibody against PKCO (Santa Cruz Biotechnology Inc.) diluted 1:100 in rinsing solution. After further washings, membranes were incubated with horseradish peroxidase-conjugated IgG fraction of goat anti-rabbit IgG (Bio-Rad) diluted 1:5,000 in PBS-Tween for 2 hours. Membrane translocation of PKC $\theta$ was expressed as the ratio of membrane bands to cytosol bands.

Tissue lipid measurement. The solid-phase extraction and purification of medium, long-chain, and very long-chain fatty acyl-CoAs from muscle has been described previously $(67,68)$. After purification, fatty acyl-CoA fractions were dissolved in methanol $/ \mathrm{H}_{2} \mathrm{O}(1: 1 \mathrm{v} / \mathrm{v})$ and subjected to liquid chromatography/mass spectrometry/mass spectrometry analysis. A turbo ionspray source was interfaced with an API 4000 tandem mass spectrometer (Applied Biosystems) in conjunction with 2 PerkinElmer 200 Series micropumps and a 200 Series autosampler (PerkinElmer). Tissue LPA content was measured as described previously (13). Approximately $70 \mathrm{mg}$ of tissue was homogenized in chloroform/methanol $(1: 1 \mathrm{v} / \mathrm{v})$ with $1 \mathrm{nmol} \mathrm{C} 17-\mathrm{LPA}$ as an internal standard. After phase separation with $\mathrm{H}_{2} \mathrm{O}$, samples were vortexed, centrifuged, and the methanol/water phase was collected. The supernatant was applied to conditioned Waters Oasis HLB extraction cartridges (Waters Corp.) and, after a washing step, LPA was eluted using methanol. Total LPA content was expressed as the sum of individual LPA species. To determine the level of DAG in cytosol and membrane, $70 \mathrm{~g}$ muscle were homogenized in buffer containing $20 \mathrm{mM}$ Tris-HCl, pH 7.4; 1 mM EDTA; 0.25 mM EGTA; $250 \mathrm{mM}$ sucrose; $2 \mathrm{mM}$ phenylmethanesulfonyl fluoride; and protease inhibitor cocktail (Roche). Samples were then centrifuged at $172,000 \mathrm{~g}$ at $4^{\circ} \mathrm{C}$ for 1 hour. The supernatants contained the cytosolic fraction, and pellets were homogenized in the above buffer again and then sonicated thoroughly. This was considered the membrane fraction. Subsequently, DAG extraction and analysis in both cytosolic and membrane samples was performed as previously described $(13,42)$. Total DAG content was expressed as the sum of individual DAG species. Tissue triglyceride was extracted using the method of Bligh and Dyer (67) and measured using a DCL Triglyceride Reagent (Diagnostic Chemicals Ltd.). Ceramide extraction and analysis were performed as previously described (42). Total ceramide contents are expressed as the sum of individual ceramide species.

AMPK activity assays. The extensor digitorum longus and tibialis anterior muscles were quickly freeze clamped in situ. Skeletal muscle samples were kept in liquid nitrogen until analyzed. Muscles were ground with a mortar and pestle and mixed with $1 \mathrm{ml}$ lysis buffer $(50 \mathrm{mM}$ Tris- $\mathrm{HCl}$ buffer, pH 7.5 at $4{ }^{\circ} \mathrm{C}$; $50 \mathrm{mM} \mathrm{NaF} ; 5 \mathrm{mM} \mathrm{NaPPi} 1 \mathrm{mM}$ EDTA; 1 mM EGTA; $1 \mathrm{mM}$ dithiothreitol; $1 \mathrm{mM}$ benzamidine; $1 \mathrm{mM}$ phenylmethanesulfonyl fluoride; $10 \% \mathrm{v} / \mathrm{v}$ glycerol; and $1 \% \mathrm{v} / \mathrm{v}$ Triton X-100). Homogenates 
were spun at $20,800 \mathrm{~g}$ for 10 minutes at $4^{\circ} \mathrm{C}$, and protein concentrations were determined. AMPK- $\alpha_{2}$ was immunoprecipitated overnight from cell lysates containing $1 \mathrm{mg}$ protein using $1 \mu \mathrm{l}$ AMPK- $\alpha_{2}$ antibody (Santa Cruz Biotechnology Inc.). Skeletal muscle AMPK- $\alpha_{2}$ activity was determined by following the incorporation of [ $\left.{ }^{32} \mathrm{P}\right] \mathrm{ATP}$ into a synthetic peptide containing the AMARA sequence on the following day.

Statistics. Analysis of variance with Duncan's multiple range post-hoc testing was used to determine significance of the difference of the means between values for RC-fed wild-type, HFD-fed wild-type, RC-fed UCP3, and HFD-fed UCP3 transgenic mice. A 2-tailed Student's $t$ test was used to determine significance of the means in all comparisons of fat-matched wild-type and UCP3 transgenic mice. Values are presented as mean \pm SEM. A $P$ value of 0.05 or less was considered significant.

\section{Acknowledgments}

The authors would like to thank John C. Clapham for kindly providing us with the UCP3 transgenic mice. This study was supported by NIH grants R01 DK-40936, P30 DK-45735, U24 DK-59635, and U24 DK-76169 (to G.I. Shulman).

Received for publication June 20, 2001, and accepted in revised form April 24, 2007.

Address correspondence to: Gerald I. Shulman, TAC S269, PO Box 9812, Yale University School of Medicine, New Haven, Connecticut 06536-9812, USA. Phone: (203) 785-5447; Fax: (203) 737-4059; E-mail: gerald.shulman@yale.edu.
1. Zimmet, P., Alberti, K.G., and Shaw, J. 2001. Global and societal implications of the diabetes epidemic. Nature. 414:782-787.

2. Shulman, G.I. 2000. Cellular mechanisms of insulin resistance. J. Clin. Invest. 106:171-176.

3. McGarry, J.D. 1992. What if Minkowski had been ageusic? An alternative angle on diabetes. Science. 258:766-770.

4. Kim, J.K., et al. 2001. Tissue-specific overexpression of lipoprotein lipase causes tissue-specific insulin resistance. Proc. Natl. Acad. Sci. U. S. A. 98:7522-7527.

5. Koyama, K., Chen, G., Lee, Y., and Unger, R.H. 1997. Tissue triglycerides, insulin resistance, and insulin production: implications for hyperinsulinemia of obesity. Am. J. Physiol. 273:E708-E713.

6. Boden, G. 1997. Role of fatty acids in the pathogenesis of insulin resistance and NIDDM. Diabetes. 46:3-10.

7. Roden, M., et al. 1996. Mechanism of free fatty acid-induced insulin resistance in humans. J. Clin. Invest. 97:2859-2865.

8. Kim, J.K., Gavrilova, O., Chen, Y., Reitman, M.L., and Shulman, G.I. 2000. Mechanism of insulin resistance in A-ZIP/F-1 fatless mice. J. Biol. Chem. 275:8456-8460.

9. Petersen, K.F., et al. 2002. Leptin reverses insulin resistance and hepatic steatosis in patients with severe lipodystrophy. J. Clin. Invest. 109:1345-1350. doi:10.1172/JCI200215001.

10. Samuel, V.T., et al. 2004. Mechanism of hepatic insulin resistance in non-alcoholic fatty liver disease. J. Biol. Chem. 279:32345-32353.

11. Samuel, V.T., et al. 2007. Inhibition of protein kinase $\mathrm{C} \varepsilon$ prevents hepatic insulin resistance in nonalcoholic fatty liver disease. J. Clin. Invest. 117:739-745. doi:10.1172/JCI30400.

12. Savage, D.B., et al. 2006. Reversal of diet-induced hepatic steatosis and hepatic insulin resistance by antisense oligonucleotide inhibitors of acetyl-CoA carboxylases 1 and 2. J. Clin. Invest. 116:817-824. doi:10.1172/JCI27300.

13. Neschen, S., et al. 2005. Prevention of hepatic steatosis and hepatic insulin resistance in mitochondrial acyl-CoA:glycerol-sn-3-phosphate acyltransferase 1 knockout mice. Cell Metab. 2:55-65.

14. Moore, L.L., et al. 2000. Can sustained weight loss in overweight individuals reduce the risk of diabetes mellitus? Epidemiology. 11:269-273.

15. Petersen, K.F., et al. 2005. Reversal of nonalcoholic hepatic steatosis, hepatic insulin resistance, and hyperglycemia by moderate weight reduction in patients with type 2 diabetes. Diabetes. 54:603-608.

16. Crawford, D., Jeffery, R.W., and French, S.A. 2000. Can anyone successfully control their weight? Findings of a three year community-based study of men and women. Int. J. Obes. Relat. Metab. Disord. 24:1107-1110.

17. Li, B., et al. 2000. Skeletal muscle respiratory uncoupling prevents diet-induced obesity and insulin resistance in mice. Nat. Med. 6:1115-1120.

18. Almind, K., Manieri, M., Sivitz, W.I., Cinti, S., and Kahn, C.R. 2007. Ectopic brown adipose tissue in muscle provides a mechanism for differences in risk of metabolic syndrome in mice. Proc. Natl. Acad. Sci. U. S. A. 104:2366-2371.

19. Mills, E.M., Banks, M.L., Sprague, J.E., and Finkel, T. 2003. Pharmacology: uncoupling the agony from ecstasy. Nature. 426:403-404.

20. Lowell, B.B., et al. 1993. Development of obesity in transgenic mice after genetic ablation of brown adipose tissue. Nature. 366:740-742.

21. Scheffler, I.E. 2001. Mitochondria make a come back. Adv. Drug Deliv. Rev. 49:3-26.

22. Kontani, Y., et al. 2005. UCP1 deficiency increases susceptibility to diet-induced obesity with age. Aging Cell. 4:147-155.

23. Borecky,J., Maia, I.G., and Arruda, P. 2001. Mitochondrial uncoupling proteins in mammals and plants. Biosci. Rep. 21:201-212.

24. Ledesma, A., de Lacoba, M.G., and Rial, E. 2002. The mitochondrial uncoupling proteins. Genome Biol. 3:REVIEWS3015.1-3015.9. doi:10.1186/gb2002-3-12-reviews3015.

25. Vidal-Puig, A., Solanes, G., Grujic, D., Flier, J.S., and Lowell, B.B. 1997. UCP3: an uncoupling protein homologue expressed preferentially and abundantly in skeletal muscle and brown adipose tissue. Biochem. Biophys. Res. Commun. 235:79-82.

26. Boss, O., et al. 1997. Tissue-dependent upregulation of rat uncoupling protein-2 expression in response to fasting or cold. FEBS Lett. 412:111-114.

27. Boss, O., et al. 1998. Uncoupling protein-3 expression in rodent skeletal muscle is modulated by food intake but not by changes in environmental temperature. J. Biol. Chem. 273:5-8.

28. Cadenas, S., et al. 1999. UCP2 and UCP3 rise in starved rat skeletal muscle but mitochondrial proton conductance is unchanged. FEBS Lett. 462:257-260.

29. Samec, S., Seydoux, J., and Dulloo, A.G. 1998. Role of UCP homologues in skeletal muscles and brown adipose tissue: mediators of thermogenesis or regulators of lipids as fuel substrate? FASEB J. 12:715-724.

30. Arsenijevic, D., et al. 2000. Disruption of the uncoupling protein-2 gene in mice reveals a role in immunity and reactive oxygen species production. Nat. Genet. 26:435-439.

31. Gong, D.W., et al. 2000. Lack of obesity and normal response to fasting and thyroid hormone in mice lacking uncoupling protein-3. J. Biol. Chem. 275:16251-16257.

32. Zhang, C.Y., et al. 2001. Uncoupling protein-2 negatively regulates insulin secretion and is a major link between obesity, beta cell dysfunction, and type 2 diabetes. Cell. 105:745-755.

33. Vidal-Puig, A.J., et al. 2000. Energy metabolism in uncoupling protein 3 gene knockout mice. J. Biol. Chem. 275:16258-16266.

34. Boss, O., et al. 1997. Uncoupling protein-3: a new member of the mitochondrial carrier family with tissue-specific expression. FEBS Lett. 408:39-42.

35. Harper, J.A., et al. 2002. Artifactual uncoupling by uncoupling protein 3 in yeast mitochondria at the concentrations found in mouse and rat skeletalmuscle mitochondria. Biochem. J. 361:49-56.

36. Samec, S., Seydoux, J., and Dulloo, A.G. 1999. Post-starvation gene expression of skeletal muscle uncoupling protein 2 and uncoupling protein 3 in response to dietary fat levels and fatty acid composition: a link with insulin resistance. Diabetes. 48:436-441.

37. Clapham, J.C., et al. 2000. Mice overexpressing human uncoupling protein-3 in skeletal muscle are hyperphagic and lean. Nature. 406:415-418.

38. Pan, D.A., et al. 1997. Skeletal muscle triglyceride levels are inversely related to insulin action. Diabetes. 46:983-988.

39. Perseghin, G., et al. 1999. Intramyocellular triglyceride content is a determinant of in vivo insulin resistance in humans: a $1 \mathrm{H}-13 \mathrm{C}$ nuclear magnetic resonance spectroscopy assessment in offspring of type 2 diabetic parents. Diabetes. 48:1600-1606.

40. Krssak, M., et al. 1999. Intramyocellular lipid concentrations are correlated with insulin sensitivity in humans: a 1H NMR spectroscopy study. Diabetologia. 42:113-116.

41. Ye, J.M., et al. 2001. Peroxisome proliferator-activated receptor (PPAR)-alpha activation lowers muscle lipids and improves insulin sensitivity in high fatfed rats: comparison with PPAR-gamma activation. Diabetes. 50:411-417.

42. Yu, C., et al. 2002. Mechanism by which fatty acids inhibit insulin activation of insulin receptor substrate-1 (IRS-1)-associated phosphatidylinositol 3-kinase activity in muscle. J. Biol. Chem. 277:50230-50236.

43. Griffin, M.E., et al. 1999. Free fatty acid-induced insulin resistance is associated with activation of protein kinase $\mathrm{C}$ theta and alterations in the insulin signaling cascade. Diabetes. 48:1270-1274.

44. Baron, A.D., Brechtel, G., Wallace, P., and Edelman, S.V. 1988. Rates and tissue sites of non-insulin- and insulin-mediated glucose uptake in humans. Am. J. Physiol. 255:E769-E774.

45. Randle, P.J., Garland, P.B., Hales, C.N., and Newsholme, E.A. 1963. The glucose fatty-acid cycle. Its role in insulin sensitivity and the metabolic disturbances of diabetes mellitus. Lancet. 1:785-789.

46. Dresner, A., et al. 1999. Effects of free fatty acids on glucose transport and IRS-1-associated phosphatidylinositol 3-kinase activity. J. Clin. Invest. 103:253-259.

47. Savage, D.B., Peteresen, K.F., and Shulman, G.I. 2007. Disordered lipid metabolism and the pathogenesis of insulin resistance. Physiol. Rev. 87:507-520.

48. Schmitz-Peiffer, C., et al. 1997. Alterations in the expression and cellular localization of protein kinase $\mathrm{C}$ isozymes epsilon and theta are associated 
with insulin resistance in skeletal muscle of the high-fat-fed rat. Diabetes. 46:169-178.

49. Previs, S.F., Withers, D.J., Ren, J.M., White, M.F., and Shulman, G.I. 2000. Contrasting effects of IRS-1 versus IRS-2 gene disruption on carbohydrate and lipid metabolism in vivo. J. Biol. Chem. 275:38990-38994.

50. Kido, Y., et al. 2000. Tissue-specific insulin resistance in mice with mutations in the insulin receptor, IRS-1, and IRS-2. J. Clin. Invest. 105:199-205.

51. Aguirre, V., Uchida, T., Yenush, L., Davis, R., and White, M.F. 2000. The c-Jun NH(2)-terminal kinase promotes insulin resistance during association with insulin receptor substrate-1 and phosphorylation of Ser(307). J. Biol. Chem. 275:9047-9054.

52. Kim, J.K., et al. 2004. PKC- $\tau$ knockout mice are protected from fat-induced insulin resistance. J. Clin Invest. 114:823-827. doi:10.1172/JCI200422230.

53. Bronfman, M., Morales, M.N., and Orellana, A. 1988. Diacylglycerol activation of protein kinase $\mathrm{C}$ is modulated by long-chain acyl-CoA. Biochem. Biophys. Res. Commun. 152:987-992.

54. Nesher, M., and Boneh, A. 1994. Effect of fatty acids and their acyl-CoA esters on protein kinase $\mathrm{C}$ activity in fibroblasts: possible implications in fatty acid oxidation defects. Biochim. Biophys. Acta.
1221:66-72.

55. Nakamura, S., and Nishizuka, Y. 1994. Lipid mediators and protein kinase $\mathrm{C}$ activation for the intracellular signaling network. J. Biochem. 115:1029-1034.

56. Thompson, A.L., Lim-Fraser, M.Y., Kraegen, E.W., and Cooney, G.J. 2000. Effects of individual fatty acids on glucose uptake and glycogen synthesis in soleus muscle in vitro. Am. J. Physiol. Endocrinol. Metab. 279:E577-E584.

57. Oakes, N.D., Cooney, G.J., Camilleri, S., Chisholm, D.J., and Kraegen, E.W. 1997. Mechanisms of liver and muscle insulin resistance induced by chronic high-fat feeding. Diabetes. 46:1768-1774.

58. Cadenas, S., et al. 2002. The basal proton conductance of skeletal muscle mitochondria from transgenic mice overexpressing or lacking uncoupling protein-3. J. Biol. Chem. 277:2773-2778.

59. Echtay, K.S., et al. 2002. Superoxide activates mitochondrial uncoupling proteins. Nature. 415:96-99.

60. Echtay, K.S., et al. 2003. A signalling role for 4hydroxy-2-nonenal in regulation of mitochondrial uncoupling. EMBO J. 22:4103-4110.

61. Abu-Elheiga, L., et al. 2000. The subcellular localization of acetyl-CoA carboxylase 2. Proc. Natl. Acad. Sci. U. S. A. 97:1444-1449.
62. Samuel, V.T., et al. 2006. Targeting foxo1 in mice using antisense oligonucleotide improves hepatic and peripheral insulin action. Diabetes. 55:2042-2050.

63. Youn, J.H., and Buchanan, T.A. 1993. Fasting does not impair insulin-stimulated glucose uptake but alters intracellular glucose metabolism in conscious rats. Diabetes. 42:757-763.

64. Alessi, D.R., et al. 1996. Mechanism of activation of protein kinase B by insulin and IGF-1. EMBO J. 15:6541-6551.

65. Folli, F., Saad, M.J., Backer, J.M., and Kahn, C.R. 1992. Insulin stimulation of phosphatidylinositol 3-kinase activity and association with insulin receptor substrate 1 in liver and muscle of the intact rat. J. Biol. Chem. 267:22171-22177.

66. Qu, X., Seale, J.P., and Donnelly, R. 1999. Tissue and isoform-selective activation of protein kinase $\mathrm{C}$ in insulin-resistant obese Zucker rats - effects of feeding. J. Endocrinol. 162:207-214.

67. Bligh, E.G., and Dyer, W.J. 1959. A rapid method of total lipid extraction and purification. Can. J. Biochem. Physiol. 37:911-917.

68. Neschen, S., et al. 2002. Contrasting effects of fish oil and safflower oil on hepatic peroxisomal and tissue lipid content. Am. J. Physiol. Endocrinol. Metab. 282:E395-E401. 